https://helda.helsinki.fi

\title{
Changing Behavior Using Self-Determination Theory
}

\section{Hagger, Martin S.}

Cambridge University Press

2020-07

Hagger , M S , Hankonen , N E \& Ryan , R M 2020 , Changing Behavior Using

Self-Determination Theory . in M S Hagger, L D Cameron, K Hamilton , N Hankonen \& T

Lintunen (eds), The Handbook of Behavior Change ., 8 , Cambridge Handbooks in

Psychology , Cambridge University Press , pp. 104-119 . https://doi.org/10.1017/9781108677318.008

http://hdl.handle.net/10138/327015

https://doi.org/10.1017/9781108677318.008

unspecified

publishedVersion

Downloaded from Helda, University of Helsinki institutional repository.

This is an electronic reprint of the original article.

This reprint may differ from the original in pagination and typographic detail.

Please cite the original version. 


\title{
8 Changing Behavior Using Self-Determination Theory
}

\author{
Martin S. Hagger, Nelli Hankonen, Nikos L. D. Chatzisarantis, \\ and Richard M. Ryan
}

\section{Practical Summary}

According to self-determination theory, it is the quality rather than the quantity of motivation that counts when it comes to behavior change. The theory distinguishes between two general classes of motivation: autonomous and controlled. People are autonomously motivated when they feel that they have freely chosen or endorsed their actions, while people are controlled motivated when they feel their actions have been dictated by others or determined by pressures or events over which they have little or no control. Beyond autonomy, the theory argues that behaviors will be better internalized and maintained when they allow satisfaction of basic psychological needs to feel competent and related to others. People who experience a behavior as autonomously motivated, and who feel competent to act, are predicted to persist with that behavior and to experience positive outcomes like interest, enjoyment, and life satisfaction as well as vitality. Studies have indicated that interventions that provide training for significant others (e.g., parents, family members, managers, teachers, coaches) to display actions and language that support the need for autonomy, as well as interventions that promote support for competence and relatedness needs, promote autonomous motivation and behavior change maintenance.

\subsection{Introduction}

Self-determination theory is a broad meta-theory that adopts a needs-based, organismic approach to understanding human behavior and attempts to understand the underlying needs and conditions within the individual that give rise to motivated behavior. In contrast to many social cognition and motivational theories (for examples, see Chapters 2

Martin S. Hagger's contribution was supported by a Finnish Distinguished Professor (FiDiPro) award from Business Finland (1801/31/2015).

https://doi.org/10.1017/9781108677318.008 and 4 , this volume), the theory offers a uniquely different approach by focusing on the quality, rather than just the quantity, of motivation as the key determinant of behavior. According to Deci and Ryan (1985b), the originators and proponents of the theory, "Cognitive theories begin their analysis with ... a motive, which is a cognitive representation of some future desired state. What is missing, of course, is the consideration of the conditions of the organism that makes these future states desired" (p. 228). The focus on the sources of one's motivation and the relations of behavior to basic psychological needs are among the key assumptions that 
make self-determination theory distinct from social cognition theories (Ryan \& Deci, 2017).

The present chapter provides an overview of the theory and research evidence on the application of self-determination theory to behavior change. The chapter begins by providing a brief overview of self-determination theory and the component mini-theories of which the broader meta-theory consists. Next, the specific ways that the theory has been used to change behavior is described, with a particular focus on autonomy support, followed by a summary of research that has applied the theory to change behavior, and tested the theory-based mechanisms responsible, in multiple behaviors, populations, and contexts. The chapter also outlines future recommendations for self-determination theory-based approaches to behavior change.

\subsection{A Brief Overview of Self- Determination Theory}

\subsubsection{Self-Determination Theory: Origins and Mini-Theories}

Self-determination theory (Deci \& Ryan, 1985b, 2000; Ryan \& Deci, 2017) is a general metatheory of motivation with origins in theories of intention, personal causation and effectance, and competence. The concept of basic psychological needs and the distinction between autonomous and controlled forms of motivation are unifying concepts central to the theory and its predictions on human motivation. The theory comprises six interconnected "mini-theories," each focusing on identifying key constructs and mechanisms that relate to particular aspects of motivation and its origins. The current chapter focuses on three of these six mini-theories that are especially pertinent to sustained behavior and behavior change: cognitive evaluation theory, organismic integration theory, and basic psychological needs theory. Accordingly, the next section introduces these three mini-theories and provides a brief overview of each, along with some key evidence supporting their predictions. The premises of these key mini-theories are also summarized in Figure 8.1 and the figure should serve as a reference guide as each mini-theory is introduced.

It is important to note that there are three additional mini-theories: causality orientations theory, goal contents theory, and relationship motivation theory. Causality orientations theory outlines how individual differences in three generalized, dispositional motivational orientations, autonomous, control, and impersonal, determine the type of motivation generally experienced by individuals across multiple behavioral domains (Deci \& Ryan, 1985a). Goal contents theory suggests that the pursuit of intrinsic and extrinsic long-term aspirations yields different effects on wellness (Vansteenkiste, Lens, \& Deci, 2006). Finally, relationship motivation theory, the newest of the mini-theories, focuses on the role of basic need support in maintaining high-quality relationships with others (Ryan \& Deci, 2017).

\subsubsection{Cognitive Evaluation Theory}

Cognitive evaluation theory concerns the concept of intrinsic motivation, a fundamental construct in self-determination theory. Intrinsic motivation is engaging in tasks or behaviors for their inherent satisfaction, without reliance on external reward contingencies or reinforcement. When individuals are intrinsically motivated to perform tasks or behaviors, they feel a sense of choice and personal effectance and derive a sense of interest, engagement, competence, and enjoyment from them. Some tasks or behaviors are inherently intrinsically motivating, such as puzzles, games, hobbies, and pastimes, but people can also become intrinsically motivated for new activities under conditions described within cognitive evaluation theory.

The extent to which individuals engage a task or behavior out of intrinsic motivation is determined, 


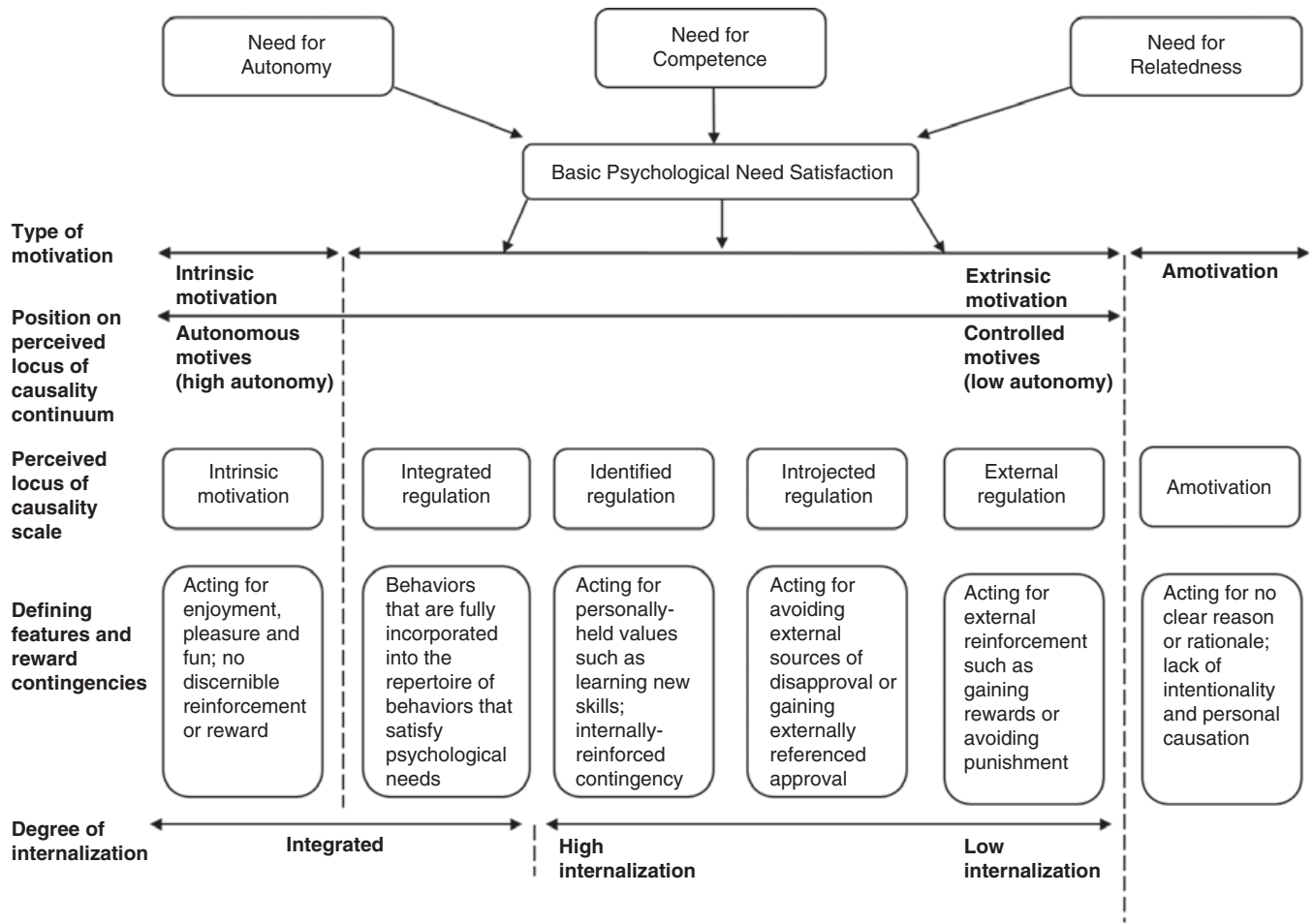

Figure 8.1 Diagram summarizing three key mini-theories of self-determination theory: Cognitive evaluation theory, organismic integration theory, and basic needs theory

to some extent, by the context in which it is performed. A key tenet of self-determination theory is that the introduction of rewards in situations where people are already intrinsically motivated can shift the individual's perception of the "cause" of their behavior from their inherent interest in the task to the external reward, undermining their intrinsic motivation (Deci, 1971). The shift in the perceived locus of causality has been studied under various types of reward contingencies, suggesting that perceptions of what is controlling one's actions can affect motivation toward, and persistence with, tasks and behaviors (Deci, Koestner, \& Ryan, 1999).

Although early research focused on the effects of varied types of rewards, cognitive evaluation theory was extended based on research suggesting that the way communications, instructions, competence feedback, deadlines, and other interpersonal events are presented similarly influences whether or not individuals' intrinsic motivation will be undermined. Specifically, communications that convey external control or pressure tend to undermine autonomy, and thus diminish intrinsic motivation, whereas those that support autonomy and feelings of competence tend to enhance intrinsic motivation. For example, Ryan, Mims, and Koestner (1983) demonstrated that, when rewards were presented as "informational" on progress rather than contingent on behavioral performance, the undermining effect was not observed. External contingencies such as criticism or controlling praise also undermined intrinsic motivation, whereas fostering choice or providing competence-related feedback enhanced intrinsic motivation. Thus, consistent with cognitive evaluation theory, the key is whether such events shift the individual's 
perceived locus of causality from internal to external or, oppositely (as in provision of choice), highlight personal causation, thereby enhancing autonomy. Research on cognitive evaluation theory paved the way for the elaborated predictions in self-determination theory and the development of other mini-theories.

\subsubsection{Organismic Integration Theory}

Organismic integration theory extends the distinction between intrinsic motivation and extrinsic forms of motivation by broadening the perceived locus of causality and outlines the processes that determine the type of motivation individuals experience when performing particular tasks or behaviors. Within organismic integration theory, perceived locus of causality was conceptualized as a continuum along which varied types of extrinsic motivation, as well as intrinsic motivation, could be located. In other words, motivational types vary in their perceived locus of causality, with some being relatively autonomous and others relatively controlled (see the top line of the continuum in Figure 8.1). At the poles of the continuum are intrinsic motivation and external regulation, two types of behavioral regulation reflecting the prototypical forms of autonomous and controlled motivation. Under intrinsic motivation, individuals view their behavior as highly volitional or autonomous, whereas in external regulation persons see their behavior as driven by externally administered rewards or punishments. Identified regulation is an autonomous form of regulation located alongside intrinsic motivation on the continuum and represents engaging in tasks or behaviors because of their perceived value or importance. In contrast, introjected regulation is located adjacent to external regulation on the continuum and reflects performing tasks and behaviors to maintain selfesteem and feel self- or other-approval. An additional form of regulation, amotivation, has also been proposed. Amotivation reflects an absence of either internal or external motives or reasons for acting and, strictly speaking, falls outside the continuum. Individuals experiencing tasks or behaviors as amotivated express listlessness, disinterest, and boredom. A person's motivation toward any given behavior can be measured experimentally or through a family of validated questionnaires, many domain- or task-specific, first proposed by Ryan and Connell (1989, see also the scale in Figure 8.1). Although it is feasible that individuals could endorse more than one regulation type for a given behavior, correlations among the constructs suggest a characteristic pattern of correlations consistent with a continuum (Chatzisarantis et al., 2003; Howard, Gagné, \& Bureau, 2017).

Organismic integration theory also proposes the processes of internalization and integration, which explain how behaviors that are not inherently engaging or interesting can come to be personally valued and maintained. Because internalization describes the process by which individuals shift their perceived locus of causality for tasks and behaviors from an external locus to an internal one, it is particularly relevant in behavior change contexts. For example, behaviors perceived as being performed for controlled reasons can be "taken in" or assimilated, leading behavior to be performed for more autonomous reasons. Integration is a complete form of internalization such that the behavior is performed for reasons that are fully congruent and self-endorsed.

Internalization can be influenced by the interpersonal context or by social agents operating in the interpersonal sphere, for example teachers in classrooms, health care staff in health consultations, leaders in organizations, or coaches in athletes' training. Deci and colleagues (1994), for instance, identified three means to promote internalization: provision of choice, providing a rationale, and acknowledging conflict. These means promote internalization by highlighting personal origin, making personally endorsed reasons for performing the behavior salient, and demonstrating social support 
by acknowledging possible challenges. These means form initial guidance on how social agents, over time, can promote internalization, and eventual integration, of behaviors, ${ }^{1}$ although there are numerous other techniques discussed within self-determination theory (Ryan \& Deci, 2017). Organismic integration theory conceptualizes internalization as a "process model," in which support for the basic needs of autonomy, competence, and relatedness by social agents fosters greater relative autonomy and thus improved behavioral persistence. Tests of the process model have demonstrated that provision of autonomy support by social agents (e.g., parents, teachers, physicians) promotes autonomous motivation and subsequent behavioral persistence, providing initial guidelines on how to change behavior based on the theory (Vasquez et al., 2015; Williams et al., 1998). A test of the self-determination theory process model is illustrated in Sidebar 8.1.

\subsubsection{Basic Needs Theory}

Basic needs theory (Deci \& Ryan, 2000) proposes that all humans have three basic or fundamental psychological needs, the fulfillment of which supports optimal functioning and wellness: needs for autonomy, competence, and relatedness. The need for autonomy reflects the need to feel that one is the "origin" of one's actions, in the sense that the person engages in them willingly and feels a sense of ownership and choice in acting. The need for competence reflects the need to feel effectance, control, and mastery over tasks and behaviors. The need for relatedness reflects the need for noncontingent, unconditional support and connectedness with others.

\footnotetext{
1 To reflect this process further, the perceived locus of causality continuum has been augmented to include integrated regulation, which reflects full internalization of tasks or behaviors that were previously controlled motivated into those that are fully integrated and experienced as autonomous (see Figure 8.1). Measures of integrated regulation, however, have not always achieved discriminant validity with other forms of regulation (Howard et al., 2017).
}

Satisfaction of these basic psychological needs, in turn, predicts optimal psychological functioning, well-being, life satisfaction, and positive affect. When needs are thwarted or frustrated, individuals experience ill-being, dissatisfaction, and negative affect, among other signs of nonoptimal functioning. A principle of complementarity means that satisfaction of all three needs is important for optimal functioning.

Persistent engagement in behaviors that are autonomously motivated is a pathway to need satisfaction. For example, behaviors that are intrinsically motivating (e.g., hobbies or pastimes), or fully internalized (e.g., pursuing tasks or behaviors that are deeply valued), are likely to fulfill all three psychological needs. For example, Weinstein and Ryan (2010) showed how volitionally engaging in helping behaviors is associated with feelings of autonomy, competence, and relatedness and explains why prosocial behaviors are so frequently associated with enhanced personal well-being. Individuals are likely to actively seek out and show greater persistence on behaviors that are need satisfying. Conversely, when individuals feel that behaviors are pressured or dictated by others (undermining autonomy), too difficult to master (undermining competence), or occur in contexts that are interpersonally unsupportive (undermining relatedness), needs are likely to be frustrated and behavior will less likely be maintained. Classic examples of needthwarting events are the imposition of unreasonable deadlines for tasks, micromanaging by leaders, or criticizing a person's competency.

\subsubsection{Putting It All Together}

Psychological need satisfaction is a unifying concept in self-determination theory and a principal mechanism that determines the type of motivation individuals experience when performing tasks or behaviors (Deci \& Ryan, 2000). Need satisfaction is also the key concept that determines how interventionists, social agents, and other leaders in the interpersonal sphere can foster positive change in 
Sidebar 8.1 Williams et al.'s (1998) test of a process model of autonomy support, autonomous motivation, and medication adherence

Williams et al. (1998) conducted a longitudinal test of a self-determination theorybased process model in predicting patients' medication adherence. Consistent with organismic integration theory and the internalization and integration processes, they proposed that patients whose physicians supported their autonomy when communicating treatment protocols would be more likely to express autonomous motivation for their treatment and report better adherence. The process model is illustrated in Figure 8.2(a).

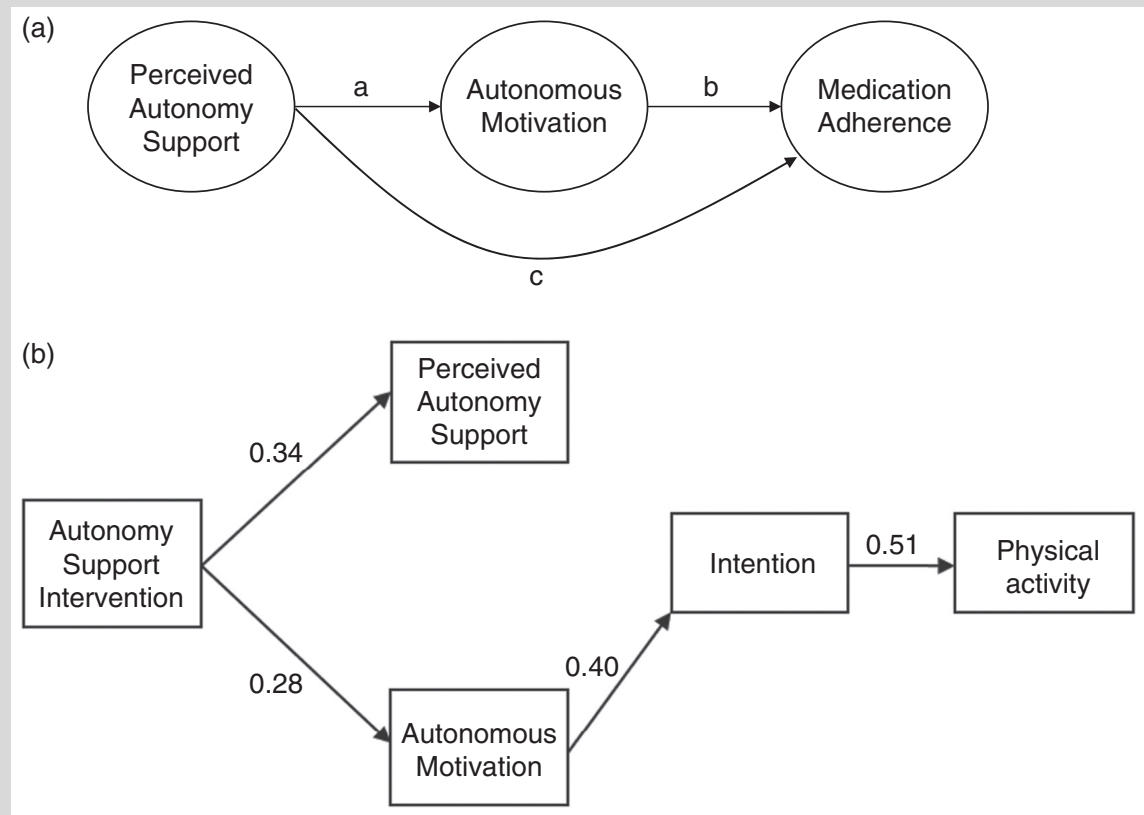

Figure 8.2 Processes in self-determination theory (a) self-determination theory process model; (b) path model showing effects of a teacher-delivered autonomy-support intervention on school children's leisure-time physical activity mediated by autonomous motivation and intention Note. Figures shown are standardized path coefficients.

Patients required to take a prescription medication for at least one month attended an interview with a clinical psychologist to discuss their health, medical regimen, relationship with their physician, and adherence. They completed self-report measures of perceived autonomy support from their physician and autonomous motivation for taking their medication. They also completed a "pill count" of their medication. Participants were contacted two weeks later and asked to conduct a follow-up pill count to measure adherence. Perceived autonomy support had a small-to-medium-sized direct effect on autonomous motivation (path a, Figure 8.2(a)), and autonomous motivation had a direct large effect on medication adherence (path b, Figure 8.2(a)). Most important, the effect of perceived autonomy support on medication adherence was fully mediated by autonomous motivation (path c, Figure 8.2(a)). This research provided an illustration that perceived support for autonomy fosters autonomous motivation and behavioral persistence. 
motivation and behavior. Individuals that perceive their behavior as need satisfying are likely to experience their actions as autonomous and are therefore likely to continue to engage in such behaviors as a means to satisfy their psychological needs, leading to behavioral persistence. Individuals that perceive their behavior as thwarting or frustrating of psychological needs are likely to experience their actions as controlled, often leading to desistence and avoidance, particularly in the absence of any continuous external contingencies or controls. If individuals persist with such behaviors, they will likely experience maladaptive outcomes such as ill-being and negative affect.

Social agents, interventionists, and other leaders may promote behavior change and maintenance by promoting autonomous motivation through autonomy support. By using strategies that support autonomy and that emphasize other potentially need-satisfying components of the behavior such as personal value, mastery, and connections with others, social agents can enhance individuals' willingness, performance, and persistence. Over time, individuals that consistently experience a behavior as need satisfying will likely internalize and integrate it into their repertoire of need-satisfying behaviors and thus experience more autonomous motivation. Generalized causality orientations, from causality orientations theory, can have a moderating effect on the extent to which communications from social agents promote internalization (Hagger \& Chatzisarantis, 2011). The minitheories of self-determination theory, therefore, provide complementary explanations of the origins of motivated behavior and outline the processes and contingencies that determine motivation, action, and persistence over time.

\subsection{How Has Self-Determination Theory Been Used to Change Behavior?}

Two key premises from self-determination theory, derived from its constituent mini-theories, provide the central pillars of strategies for behavior change interventions: autonomous motivation and basic psychological need support. According to the theory, autonomous motivation and need satisfaction can be fostered using autonomy- and need-supportive strategies, typically communicated to the target population by social agents, as well as by the strategic structuring of feedback and contingencies. Autonomy- and need-supportive strategies highlight autonomous reasons for participating in the behavior of interest or value, provide choices where possible, make the need-satisfying features of the behavior more salient to the individual (e.g., by indicating choice or providing a personally relevant rationale), and provide opportunities for individuals to experience tasks and behaviors as consistent with their needs, values, and motives. A growing number of studies has tested the efficacy of interventions and programs adopting autonomy- and need-supportive strategies in changing behavior (see $\mathrm{Ng}$ et al., 2012). While interventions are typically delivered by social agents in face-toface situations, they have also been communicated by written messages such as online and smartphone-based interventions. In the next section the form and content of self-determination theory-based interventions are introduced, and research examining the efficacy of self-determination theory-based interventions in changing behavior summarized.

\subsubsection{Need Support}

Autonomy-supportive strategies include not only provision of choice but also a meaningful rationale that can provide a basis for volitional engagement. In addition, supportive interventions scaffold tasks so that people can feel a sense of growth and mastery (supporting need for competence). Finally, interventions that are interpersonally supportive (supporting the need for relatedness) lead to more willingness to connect and internalize behavior change. Thus, acknowledging the person's 
perspective and experiences can enhance behavioral maintenance.

Research focused on promoting internalization has indicated that a combination of several strategies is most effective in moving individuals to being more autonomously motivated by highlighting to the individual that their actions are freely chosen, that they have a personal reason or rationale for doing the behavior, and that the social agent recognizes the challenge presented by the behavior and therefore communicates a sense of understanding and regard for the individual in performing the behavior (Ryan \& Deci, 2017).

These formulations have been supported by a substantial literature. For example, experimental studies have confirmed the role of choice as a fundamental component of autonomy-support interventions (Patall, Cooper, \& Robinson, 2008). In addition, research in the field of education has identified the autonomy-supportive strategies that teachers adopt to promote autonomous motivation and behavior change among students (Deci et al., 1982; Reeve, Bolt, \& Cai, 1999; Reeve \& Jang, 2006). These findings are based on formative research demonstrating consistent links between autonomous forms of motivation and school students' interest and engagement in class and academic attainment (Reeve, 2002). The research has focused on the kinds of behaviors teachers display in class ("what teachers do") and the language content and style when they instruct students ("what teachers say") that communicate and foster autonomous motivation among students (Reeve \& Jang, 2006; Reeve et al., 2004). Research has also identified the controlling behaviors and language that undermine psychological needs and lead to maladaptive outcomes (Deci et al., 1982). Minimizing the use of controlling behaviors is also important to promote autonomous motivation (Tilga et al., 2019).

Reeve and colleagues $(2002,2006)$ conducted an influential set of studies that identified the autonomy-supportive and controlling behaviors that teachers typically adopt in classroom contexts (see also Chapter 35, this volume). The research is a preeminent example of structured means to identify, code, and assess these kinds of behaviors in teachers, which may inform the content of behavior change interventions based on self-determination theory. They developed a list of twenty-one autonomy-supportive and controlling teacher behaviors, based on previous research that had observed and manipulated teachers' behaviors from a self-determination theory perspective (Deci et al., 1982; Reeve et al., 1999). They coded the instructional behaviors used by a sample of teachers during a ten-minute teaching interaction and measured the perceived autonomy, engagement, and performance of their students. Autonomy-supportive (e.g., time listening; time student talking; communicating perspectivetaking statements; time allowing student to work in own way; providing informational feedback; offering encouragement; offering hints; being responsive to students' questions) and controlling (e.g., exhibiting solutions/answers; uttering solutions/answers; time holding/monopolizing learning materials; uttering directives/commands; making should/got to statements; asking controlling questions) behaviors were consistently associated with autonomous motivation and learning ratings for the students. This research identified the behaviors autonomy-supportive teachers would expect to display in order to promote students' autonomous motivation and adaptive outcomes in class. It also has significant translational value by providing a template for the identification of behaviors that social agents in other contexts may use to support autonomy. The list of behaviors is presented in Appendix 8.1 (supplemental materials) and includes operational definitions of each.

A leading approach to developing effective autonomy-support interventions in education contexts is autonomy-support training programs (Cheon \& Reeve, 2013; Cheon, Reeve, \& Moon, 2012; Reeve \& Jang, 2006; see 
Chapter 35, this volume). These programs recognize the importance of training teachers to display autonomy-supportive behaviors and use autonomy-supportive language when communicating with students. In essence, the approach recognizes the need to change the behavior of those delivering the intervention in order to change the behavior of the target populations (see also Chapter 21, this volume). Promoting autonomy support in educational settings requires teachers to adopt and consistently apply sets of autonomy-supportive behaviors in their everyday lessons. The training programs provide instructional materials and teaching plans to develop teachers' competencies in the use of these behaviors. The programs include descriptions of the autonomy-supportive behaviors, teaching points and examples of the use of the behaviors, tips on how to develop the behaviors, and relevant practices that teachers can perform to build competency in their use. Meta-analyses have demonstrated that autonomy-support training programs are generally effective in changing teachers' behavior (Su \& Reeve, 2011).

Interventions that promote competence and relatedness need support have also been explored. Research has demonstrated that the provision of mastery experiences promotes autonomous motivation by enhancing competence (e.g., Jang, Reeve, \& Deci, 2010; Tessier, Sarrazin, \& Ntoumanis, 2010). Interventions aimed at promoting competence may play a role in enhancing autonomous motivation. Similarly, research has also identified behaviors that promote relatedness need support and their effects on autonomous motivation and behavioral engagement (e.g., Sparks et al., 2017). However, providing support for competence and relatedness needs in the absence of autonomy support may not be sufficient to promote internalization of behaviors and autonomous motivation. For example, it is possible for individuals to feel competent in performing tasks but to not feel they have complete ownership over their actions because they view their behavior as controlled by external forces. Individuals can, therefore, feel competent but not autonomous. Furthermore, there may be interplay between need-supportive behaviors. For example, support for autonomy has also been shown to foster competence (Williams, Lynch, \& Glasgow, 2007). More research is needed to systematically examine the extent to which different types of need support also support autonomous motivation and internalization.

\subsubsection{Motivation and Behavior Change Techniques}

Following research identifying need-supportive behaviors in education (Reeve, 2002; Reeve et al., 1999; Reeve \& Jang, 2006), Teixeira and colleagues (2020) conducted a study to develop a comprehensive description of the strategies or techniques used to promote autonomous motivation and behavior change based on self-determination theory in health contexts. Psychological need support was used as a central organizing principle. The researchers identified a list of candidate need-supportive techniques from a comprehensive literature review. They then matched each technique with its "primary" psychological need and produced labels, definitions, and functional descriptions for each. Next, the list of candidate techniques was circulated to a group of self-determination theory experts, who rated each technique according to its uniqueness, redundancy, essentiality, and match with its primary psychological need. Using a consensus approach from the expert ratings, a classification of twenty-one techniques was developed.

The techniques identified from the consensus study are presented in Appendix 8.2 (supplemental materials), classified according to its primary targeted need. Although some of the techniques are common to those identified in previous research (e.g., Reeve \& Jang, 2006), the classification is the first attempt to comprehensively isolate the unique techniques that comprise self-determination 
theory interventions. The classification will function as a resource for researchers and practitioners to inform the content of behavior change interventions; provide guidance on how those techniques might "work" in promoting need satisfaction, autonomous motivation, and behavior change; and deliver a common set of terms and descriptions to help researchers accurately report the content of self-determination theory interventions. Although the classification has been developed through consensus, future research is needed to establish its validity by using it as a basis for rigorous tests of selfdetermination theory-based interventions.

\subsubsection{Evidence for Self-Determination Theory Interventions}

There is a considerable body of research on the efficacy of interventions based on selfdetermination theory in promoting motivation and changing behavior across multiple disciplines, populations, and behaviors (e.g., Chatzisarantis \& Hagger, 2009; Cheon \& Reeve, 2013; Silva et al., 2010). Interventions typically adopt combinations of the autonomy-supportive strategies or seek to minimize or eliminate controlling strategies displayed by social agents to "create" an autonomysupportive environment to promote the behavior of interest to the target population. Some interventions have delivered autonomy-supportive interventions via print communication or media such as websites or mobile phone apps (e.g., Spring et al., 2013). Studies evaluating self-determination theory-based interventions have tended to use controlled designs, with some using fully randomized controlled, experimental, or longitudinal pre- and post-intervention designs (e.g., Chatzisarantis \& Hagger, 2009; Cheon et al., 2012; Hankonen et al., 2016; Shah et al., 2016; Silva et al., 2010; Williams et al., 2006). Studies usually use trained facilitators as the "social agents" that deliver the intervention content or train existing social agents to use the techniques, using an autonomy-support training program or similar. For example, Williams et al. (2006) trained counselors to support smokers in making autonomous decisions to quit, and Cheon et al. (2012) trained teachers to display autonomy-supportive behaviors using their autonomy-support training program. Interventions are usually evaluated using relevant outcome measures of the behavior of interest at single or multiple follow-up occasions after the initiation of the intervention (e.g., Williams et al., 2016).

Importantly, researchers have also included measures of key theoretical constructs expected to change as a consequence of the intervention. These measures include autonomous and controlled forms of motivation toward the behavior of interest, psychological need satisfaction, interest, enjoyment, competence, life satisfaction, and vitality. Measures such as autonomous motivation and need satisfaction are implicated in the process by which the intervention changes behavior consistent with the theory. These are considered mediators of the effects of the interventions, such that they explain "how" the content of the intervention results in behaviors change. Measures such as interest, enjoyment, and vitality are expected outcomes of engaging in a behavior for autonomous reasons and when needs are satisfied and are therefore considered secondary outcomes. Consistent with the designs of studies evaluating self-determination theory interventions, measures of behavioral and motivational outcomes are typically measured at multiple time points in conjunction with behavioral measures to establish the effects of intervention in changing outcomes over time. Such evaluations involve testing the extent to which measures of the self-determination theory constructs, such as perceived satisfaction of psychological needs and autonomous motivation, mediate the effect of the intervention on the behavioral outcome measured at post-intervention follow-up (e.g., Chatzisarantis \& Hagger, 2009; Silva et al., 2010; Williams et al., 2006). An illustrative example is presented in Sidebar 8.2. 
Sidebar 8.2 Chatzisarantis and Hagger's (2009) process evaluation of an autonomy-support intervention

Chatzisarantis and Hagger developed an intervention to promote physical activity among high school students outside of school in their "leisure time." Their intervention adopted an autonomy-support training program (see Cheon \& Reeve, 2013; Cheon et al., 2012; Reeve et al., 2004) to promote teachers' use of autonomysupportive behaviors in their lessons to promote autonomous motivation toward the target behavior in their students. Teachers assigned to an autonomy-support intervention condition received training on autonomy-supportive behaviors (e.g., providing positive feedback, giving a rationale, acknowledging difficulties, enhancing choice). Teachers assigned to a control condition were provided training that did not include the autonomy-supportive components. After training, teachers implemented their training in their regular lessons for five weeks. Students' autonomous motivation, perceived autonomy support, leisure-time physical activity intentions, and leisure-time physical activity participation were measured pre- and post-intervention. Path analysis tested the effects of the intervention on follow-up measures and leisure-time physical activity participation. Results revealed small-tomedium-size direct effects of the intervention on students' autonomous motivation and perceived autonomy support, a medium-size direct effect of autonomous motivation on intentions, a medium-size direct effect of intentions on physical activity participation, and a small-size indirect effect of the intervention through autonomous motivation and intentions. Findings are summarized in Figure 8.2(b). These illustrate the efficacy of the intervention in changing behavior, as well as the processes by which the intervention is presumed to affect behavior change.

These effects are a specific example of the more generalized process model of how autonomy- and need-supportive interventions impact behavior change and associated outcomes through changes in need satisfaction and autonomous motivation (see Fortier et al., 2012 and the figure presented in Appendix 8.3, supplemental materials).

A growing body of research demonstrates the efficacy of autonomy- and need-support interventions in promoting motivation and behavior change and adaptive outcomes in health (e.g., Gillison et al., 2018; Ng et al., 2012; Teixeira, Palmeira, \& Vansteenkiste, 2012), occupational (e.g., Deci, Connell, \& Ryan, 1989), and educational (e.g., Cheon et al., 2012; Reeve et al., 2004) contexts. When evaluating the efficacy of these interventions, it is important to consider that most interventions aim to promote changes in autonomous motivation and behavior of the target population (e.g., students, employees), by promoting autonomy- and need-supportive behaviors in appropriate social agents (e.g., teachers, managers). In many contexts (e.g., schools, workplaces), this means changing the behavior of the social agents themselves. Some interventions, therefore, have the end goal of changing the behavior of the teachers themselves (Cheon \& Reeve, 2013; Su \& Reeve, 2011). For example, a meta-analysis in educational contexts has shown autonomy-supportive interventions to be effective in producing change in teachers' use of 
autonomy-supportive training styles, with longerterm interventions more effective ( $\mathrm{Su} \&$ Reeve, 2011). However, most aim to achieve change in the target population or both. It is also important to account for the goal of the intervention when assessing its efficacy, often illustrated by change in the dependent variables. A key question, therefore, is whether the intervention focuses on changing the autonomy-supportive behaviors of the social agents alone or both the social agents and the target population.

Systematic reviews and meta-analyses have illustrated consistent effects of autonomy- and need-support interventions on individuals' behavior in particular domains. For example, Teixeira and colleagues (2012) demonstrated that the majority of studies adopting autonomysupportive interventions found effects on physical activity participation and theoretical constructs, including perceived autonomy support, need satisfaction, and autonomous motivation. Similarly, $\mathrm{Ng}$ et al.'s (2012) meta-analysis revealed small-to-medium-size overall averaged effects of autonomous motivation on health behavior participation among studies adopting experimental and intervention designs, and these effects were stronger than effects in studies adopting nonexperimental designs. Overall, the evidence for autonomy-support interventions seems to be consistent, although, to date, there has been no quantitative research synthesis of autonomy- and need-support interventions on motivational and behavioral outcomes across multiple behavioral domains.

The majority of autonomy-support interventions have evaluated change over a relatively brief period, with follow-up measures of behavior change being only a few weeks post-intervention (Cheon \& Reeve, 2013; Ng et al., 2012). However, there is some evidence of long-term intervention effects over a year or more post-intervention (e.g., Cheon \& Reeve, 2013; Silva et al., 2010). For example, Cheon and Reeve (2013) demonstrated substantive effects of their autonomy-supportive intervention on children's autonomous motivation, academic attainment, and adaptive outcomes in school physical education over a one-year period. Similarly, Silva et al. (2010) demonstrated maintenance of the effects of a thirty-session facilitatorled autonomy-support intervention on physical activity participation, weight-loss outcomes, and self-determination theory one and two years postintervention. Although the research evidence is relatively sparse and confined to the health domain, autonomy-support interventions demonstrate considerable promise in fostering long-term motivation and behavior change.

Autonomy-support interventions vary in their duration and intensiveness, or "dose," usually determined by intervention duration and the "contact time" that participants spend with the social agents delivering the intervention. While some brief interventions have been effective (e.g., Chatzisarantis \& Hagger, 2009; Tessier et al., 2010), there is evidence that more intensive interventions involving long-term and frequent exposures to autonomy-supportive strategies tend to be more effective. Teixeira et al. (2012) noted considerable variability in the duration of self-determination theory-based interventions used to promote physical activity and noted that most were less than three months in duration and involved only a brief amount of contact time. They also noted considerable variability in the numbers of strategies used and the extent to which the interventions were based on the theory. However, they did not note whether this variability in content coincided with variability in efficacy. To date, there is no study that has systematically varied the duration and dose of self-determination theory interventions and assessed their effects on motivation and behavior change, and this remains an important avenue for future research.

Many self-determination theory-based interventions comprise multiple techniques in a single intervention and test intervention effects on behavior change relative to a no-intervention control 
group. In contrast, few studies have tested effects of individual autonomy-support intervention techniques on behavior change using factorial designs. The development of the classification of motivational and behavior change techniques provides a useful starting point for testing the main, additive, and interactive effects of individual techniques on motivation and behavior change (Teixeira et al., 2020). Research that systematically evaluates the effects of different techniques on motivation mediators (e.g., autonomous motivation, need satisfaction) and behavior change will progress knowledge by identifying the most effective techniques, as well as those that are less effective or redundant. It will also assist in identifying combinations of intervention techniques that lead to greater behavior change than each of the component techniques alone.

\subsection{Conclusion and Future Directions}

Self-determination theory is a generalized theory of human motivation and wellness, with the quality of motivation, psychological need satisfaction, and environmental supports for motivation as key components. The theory proposes a fundamental distinction between autonomous motivation, characterized by reasons for acting that are selfendorsed and experienced as volitional, and controlled motivation, defined as acting out of externally-referenced reasons and experienced as determined by events or pressures outside the self. Autonomous forms of motivation have been consistently linked to better persistence with behaviors and more adaptive outcomes. Individuals' motivational quality is influenced by the extent to which basic psychological needs for autonomy, competence, and relatedness are supported and satisfied.

Behavior change interventions based on selfdetermination theory have focused on providing support for psychological needs to promote autonomous motivation and behavioral persistence. Most interventions focus on training social agents (e.g., teachers, managers) to provide support for the autonomy of the target population. Research has demonstrated the efficacy of interventions based on the theory in changing behavior and theory-based constructs, including autonomous motivation, perceived autonomy support, and adaptive outcomes. Process evaluations of interventions have also demonstrated the role of autonomous motivation in mediating intervention effects on behavior change.

Recent research has developed a classification of the techniques that comprise need-supportive interventions (Teixeira et al., 2020). Future research may seek to examine the unique and interactive effects of specific techniques on behavior change and provide further evidence of longterm effectiveness of interventions based on the theory. Extending this idea, a system to characterize motivating and demotivating style along the dimensions of provision of need support and level of directiveness has recently been developed (Aelterman et al., 2019). Four "styles" were identified, each defined by a quadrant bounded by high and low levels of the need-supportive and directiveness dimensions: autonomy-supportive, structuring, controlling, and chaotic. Future research should seek to establish the motivational and behavioral consequences of social agents adopting each style. Finally, research has also examined whether individuals can apply autonomy-supportive strategies to motivate their own behavior. Such self-enactable strategies may include modifying the behavior or task to be more enjoyable, reminding oneself of personally important reasons to engage in the behavior, aligning one's identity with the target behavior, modifying ways of doing the behavior to allow for participation in other valued behaviors or pursuit of valued outcomes, reflecting on autonomous goals, and reminding oneself of past success in the behavior (Knittle et al., 2020). Research is needed to explore the effectiveness of these strategies in enabling individuals to change their own behavior. 


\section{References}

Aelterman, N., Vansteenkiste, M., Haerens, L., Soenens, B., Fontaine, J. R. J., \& Reeve, J. (2019). Toward an integrative and fine-grained insight in motivating and demotivating teaching styles: The merits of a circumplex approach. Journal of Educational Psychology, 111, 497-521. https:// doi.org/10.1037/edu0000293

Chatzisarantis, N. L. D., \& Hagger, M. S. (2009). Effects of an intervention based on selfdetermination theory on self-reported leisure-time physical activity participation. Psychology and Health, 24, 29-48. https://doi.org/10.1080/ 08870440701809533

Chatzisarantis, N. L. D., Hagger, M. S., Biddle, S. J. H., Smith, B., \& Wang, C. K. J. (2003). A meta-analysis of perceived locus of causality in exercise, sport, and physical education contexts. Journal of Sport and Exercise Psychology, 25, 284-306. https://doi.org/ 10.1123/jsep.25.3.284

Cheon, S. H., \& Reeve, J. (2013). Do the benefits from autonomy-supportive PE teacher training programs endure? A one-year follow-up investigation. Psychology of Sport and Exercise, 14, 508-518. https://doi.org/10.1016/ j.psychsport.2013.02.002

Cheon, S. H., Reeve, J., \& Moon, I. (2012). Experimentally based, longitudinally designed, teacher-focused intervention to help physical education teachers be more autonomy supportive toward their students. Journal of Sport and Exercise Psychology, 34, 365-396. https://doi.org/ 10.1123/jsep.34.3.365

Deci, E. L. (1971). Effects of externally mediated rewards on intrinsic motivation. Journal of Personality and Social Psychology, 18, 105-115.

Deci, E. L., Connell, J. P., \& Ryan, R. M. (1989). Selfdetermination in a work organization. Journal of Applied Psychology, 74, 580-590. https://doi.org/ 10.1037/0021-TS (global): Change en-dashes in URLs for DOIs to hyphens throughout to ensure links work correctly.9010.74.4.580

Deci, E. L., Eghrari, H., Patrick, B. C., \& Leone, D. R. (1994). Facilitating internalization: The selfdetermination theory perspective. Journal of
Personality, 62, 119-142. https://doi.org/10.1111/ j.1467-6494.1994.tb00797.x

Deci, E. L., Koestner, R., \& Ryan, R. M. (1999). A meta-analytic review of experiments examining the effects of extrinsic rewards on intrinsic motivation. Psychological Bulletin, 125, 627668.

Deci, E. L., \& Ryan, R. M. (1985a). The general causality orientations scale: Self-determination in personality. Journal of Research in Personality, 19, 109-134. https://doi.org/10.1016/0092-6566 (85)90023-6

Deci, E. L., \& Ryan, R. M. (1985b). Intrinsic Motivation and Self-Determination in Human Behavior. New York: Plenum Press.

Deci, E. L., \& Ryan, R. M. (2000). The "what" and "why" of goal pursuits: Human needs and the selfdetermination of behavior. Psychological Inquiry, 11, 227-268. https://doi.org/10.1207/ S15327965PLI1104 01

Deci, E. L., Spiegel, N. H., Ryan, R. M., Koestner, R., \& Kauffman, M. (1982). Effects of performance standards on teaching styles: Behavior of controlling teachers. Journal of Educational Psychology, 74, 852-859. https://doi.org/10.1037/ 0022-0663.74.6.852

Fortier, M. S., Duda, J. L., Guerin, E., \& Teixeira, P. J. (2012). Promoting physical activity: Development and testing of self-determination theory-based interventions. International Journal of Behavioral Nutrition and Physical Activity, 9, 20. https://doi .org/10.1186/1479-5868-9-20

Gillison, F. B., Rouse, P., Standage, M., Sebire, S., \& Ryan, R. M. (2018). A meta-analysis of techniques to promote motivation for health behaviour change from a self-determination theory perspective. Health Psychology Review, 13, 110-130. https://doi.org/10.1080/ 17437199.2018.1534071

Hagger, M. S., \& Chatzisarantis, N. L. D. (2011). Causality orientations moderate the undermining effect of rewards on intrinsic motivation. Journal of Experimental Social Psychology, 47, 485-489. https://doi.org/10.1016/j.jesp.2010.10.010

Hankonen, N., Heino, M. T. J., Araujo-Soares, V. et al. (2016). "Let's Move It": A school-based multilevel intervention to increase physical 
activity and reduce sedentary behaviour among older adolescents in vocational secondary schools: A study protocol for a cluster-randomised trial. BMC Public Health, 16, 451. https://doi.org/ 10.1186/s12889-016-3094-x

Howard, J. L., Gagné, M., \& Bureau, J. S. (2017). Testing a continuum structure of self-determined motivation: A meta-analysis. Psychological Bulletin, 143, 1346-1377. https://doi.org/ $10.1037 /$ bul0000125

Jang, H., Reeve, J., \& Deci, E. L. (2010). Engaging students in learning activities: It is not autonomy support or structure but autonomy support and structure. Journal of Educational Psychology, 102, 588-600. https://doi.org/10.1037/a0019682

Knittle, K., Heino, M. T. J., Marques, M. M., Stenius, M., Beattie, M., Ehbrecht, F., Hagger, M. S., Hardeman, W., \& Hankonen, N. (2020). The compendium of self-enactable techniques to change and self-manage motivation and behaviour v1. 0. Nature Human Behavior, 4, 215-223. https://doi.org/10.1038/s41562-019-0798-9.

Ng, J. Y. Y., Ntoumanis, N., Thøgersen-Ntoumani, C. et al. (2012). Self-determination theory applied to health contexts. Perspectives on Psychological Science, 7, 325-340. https://doi.org/10.1177/ 1745691612447309

Patall, E. A., Cooper, H., \& Robinson, J. C. (2008). The effects of choice on intrinsic motivation and related outcomes: A meta-analysis of research findings. Psychological Bulletin, 134, 270-300. https://doi.org/10.1037/0033-2909.134.2.270

Reeve, J. (2002). Self-determination theory applied to educational settings. In E. L. Deci \& R. M. Ryan (Eds.), Handbook of Self-Determination Research (pp. 183-203). Rochester, NY: University of Rochester Press.

Reeve, J., Bolt, E., \& Cai, Y. (1999). Autonomysupportive teachers: How they teach and motivate students. Journal of Educational Psychology, 91, 537-548. https://doi.org/10.1037/00220663.91 .3 .537

Reeve, J., \& Jang, H. (2006). What teachers say and do to support students' autonomy during a learning activity. Journal of Educational Psychology, 98, 209-218. https://doi.org/10.1037/00220663.98.1.209
Reeve, J., Jang, H., Carrell, D., Jeon, S. Y., \& Barch, J. (2004). Enhancing students' engagement by increasing teachers' autonomy support. Motivation and Emotion, 28, 147-169. https://doi .org/10.1023/B:MOEM.0000032312.95499.6 f

Reeve, J., Jang, H., Hardre, P., \& Omura, M. (2002). Providing a rationale in an autonomy-supportive way as a strategy to motivate others during an uninteresting activity. Motivation and Emotion, 26, 183-207. https://doi.org/10.1023/ a: 1021711629417

Ryan, R. M., \& Connell, J. P. (1989). Perceived locus of causality and internalization: Examining reasons for acting in two domains. Journal of Personality and Social Psychology, 57, 749-761. https://doi .org/10.1037/0022-3514.57.5.749

Ryan, R. M., \& Deci, E. L. (2017). Self-Determination Theory: Basic Psychological Needs in Motivation, Development and Wellness. New York: Guilford Press.

Ryan, R. M., Mims, V., \& Koestner, R. (1983). Relation of reward contingency and interpersonal context to extrinsic motivation: A review and test using cognitive evaluation theory. Journal of Personality and Social Psychology, 45, 736-750.

Shah, K. N., Majeed, Z., Yoruk, Y. B. et al. (2016). Enhancing physical function in HIV-infected older adults: A randomized controlled clinical trial. Health Psychology, 35, 563-573. https://doi .org/10.1037/hea0000311

Silva, M. N., Vieira, P. N., Coutinho, S. R. et al. (2010) Using self-determination theory to promote physical activity and weight control: A randomized controlled trial in women. Journal of Behavioral Medicine, 33, 110-122. https://doi .org/10.1007/s10865-009-9239-y

Sparks, C., Lonsdale, C., Dimmock, J. A., \& Jackson, B. (2017). An intervention to improve teachers' interpersonally involving instructional practices in high school physical Education: Implications for student relatedness support and in-class experiences. Journal of Sport and Exercise Psychology, 39, 120-133. https://doi.org/10.1123/ jsep.2016-0198

Spring, B., Gotsis, M., Paiva, A., \& Spruijt-Metz, D. (2013). Healthy apps: Mobile devices for continuous monitoring and intervention. IEEE 
Pulse, 4, 34-40. https://doi.org/10.1109/

MPUL.2013.2279620

Su, Y. L., \& Reeve, J. (2011). A meta-analysis of the effectiveness of intervention programs designed to support autonomy. Educational Psychology Review, 23, 159-188. https://doi.org/10.1007/ s10648-010-9142-7

Teixeira, P. J., Palmeira, A., \& Vansteenkiste, M. (2012). The role of self-determination theory and motivational interviewing in behavioral nutrition, physical activity, and health: An introduction to the IJBNPA special series. International Journal of Behavioral Nutrition and Physical Activity, 9, 17. https://doi.org/10.1186/1479-5868-9-17

Teixeira, P. J., Marques, M. M., Silva, M. N. et al. (2020). Classification of techniques used in selfdetermination theory-based interventions in health contexts: An expert consensus study. Motivation Science. https://doi.org/10.1037/mot0000172

Tessier, D., Sarrazin, P., \& Ntoumanis, N. (2010). The effect of an intervention to improve newly qualified teachers' interpersonal style, students motivation and psychological need satisfaction in sport-based physical education. Contemporary Educational Psychology, 35, 242-253. https://doi .org/10.1016/j.cedpsych.2010.05.005

Tilga, H., Hein, V., Koka, A., Hamilton, K., \& Hagger, M. S. (2019). The role of teachers' controlling behaviour in physical education on adolescents' health-related quality of life: Test of a conditional process model. Educational Psychology, 39, 862880. https://doi.org/10.1080/

01443410.2018 .1546830

Vansteenkiste, M., Lens, W., \& Deci, E. L. (2006). Intrinsic versus extrinsic goal contents in selfdetermination theory: Another look at the quality of academic motivation. Educational
Psychologist, 41, 19-31. https://doi.org/10.1207/ s15326985ep4101_4

Vasquez, A. C., Patall, E. A., Fong, C. J., Corrigan, A. S., \& Pine, L. (2015). Parent autonomy support, academic achievement, and psychosocial functioning: A meta-analysis of research. Educational Psychology Review, 28, 605-644. https://doi.org/10.1007/s10648-015-9329-z

Weinstein, N., \& Ryan, R. M. (2010). When helping helps: Autonomous motivation for prosocial behavior and its influence on well-being for the helper and recipient. Journal of Personality and Social Psychology, 98, 222-244. https://doi.org/ 10.1037/a0016984

Williams, G. C., Deci, E. L., Niemiec, C. P., Ryan, R. M., \& Patrick, H. (2016). Outcomes of the smoker's health project: A pragmatic comparative effectiveness trial of tobacco-dependence interventions based on self-determination theory. Health Education Research, 31, 749-759. https:// doi.org/10.1093/her/cyw046

Williams, G. C., Lynch, M., \& Glasgow, R. E. (2007). Computer-assisted intervention improves patientcentered diabetes care by increasing autonomy support. Health Psychology, 26, 728-734. https:// doi.org/10.1037/0278-6133.26.6.728

Williams, G. C., McGregor, H. A., Sharp, D. et al. (2006). Testing a self-determination theory intervention for motivating tobacco cessation: Supporting autonomy and competence in a clinical trial. Health Psychology, 25, 91-101. https://doi.org/10.1037/0278-6133.25.1.91

Williams, G. C., Rodin, G. C., Ryan, R. M., Grolnick, W. S., \& Deci, E. L. (1998). Autonomous regulation and long-term medication adherence in adult outpatients. Health Psychology, 17, 269276 . 